\title{
Géographie du Paludisme dans la région de Libreville-Owendo-Akanda, Gabon
}

\author{
Alexis Mbouloungou, \\ Laboratoire de Géomatique, de Recherche Appliquée et de Conseil \\ (LAGRAC), Université Omar Bongo (UOB), Faculté des Lettres et Sciences \\ Humaines (FLSH), Libreville, Gabon

\section{Aubin Armel Koumba,} \\ Laboratoire d'Ecologie Vectorielle (LEV), Institut de Recherche en Ecologie \\ Tropicale (IRET), Centre National de la Recherche Scientifique et \\ Technologique, Libreville, Gabon \\ Faculté des Sciences et Techniques (FAST), \\ Université d'Abomey-Calavi, Cotonou, Bénin
}

\section{Jean Bernard Mombo,}

Laboratoire d'Analyse Spatiale et des Environnements Tropicaux (LANASPET), Université Omar Bongo (UOB), Faculté des Lettres et

Sciences Humaines (FLSH), Libreville, Gabon

\section{Nadine Ndonghan Iyangui,}

Laboratoire de Géomatique, de Recherche Appliquée et de Conseil (LAGRAC), Université Omar Bongo (UOB), Faculté des Lettres et Sciences Humaines (FLSH), Libreville, Gabon

\section{Jacques François Mavoungou,}

Laboratoire d'Ecologie Vectorielle (LEV), Institut de Recherche en Ecologie

Tropicale (IRET), Centre National de la Recherche Scientifique et

Technologique, Libreville, Gabon

\section{Jules Djeki,}

Laboratoire de Géomatique, de Recherche Appliquée et de Conseil

(LAGRAC), Université Omar Bongo (UOB), Faculté des Lettres et Sciences

Humaines (FLSH), Libreville, Gabon

Doi:10.19044/esj.2019.v15n27p362 URL:http://dx.doi.org/10.19044/esj.2019.v15n27p362

\section{Resumé}

Afin de déterminer la géographie du paludisme, de connaître la distribution spatiale des formations sanitaires et d'identifier les zones à risque palustre dans la region de Libreville-Owendo-Akanda, une étude a été menée de juillet 2016 à juin 2017. Cette étude a consisté à récolter les données de morbidité palustre hospitalière et à caractériser les structures sanitaires 
publiques et privées de ladite zone. Les données de paludisme ont été collectées dans les cliniques au moyen des fiches de collecte pré-conçues et testées. De plus, la cartographie des structures de santé et des chefferies des différents quartiers, d'où provenait chaque malade, s'est faite à l'aide d'un GPS-MAP 62s de marque Garmin. Des analyses spatiales ont été faites dans ArcGIS 10.3 selon le modèle des Hot Spots, pour identifier les zones à risques du paludisme. Ainsi, 211 structures privées et publiques de santé reparties en 12 types ont été répertoriées. Ces structures sont inégalement réparties dans la région de Libreville-Owendo-Akanda. Les mieux représentés sont les cabinets médicaux privés $(\mathrm{n}=62 ; 29,38 \%)$, les pharmacies $(\mathrm{n}=39 ; 18,48 \%)$, les cabinets des soins infirmiers $(\mathrm{n}=36 ; 17,06 \%)$. Tandis que les moins représentés sont les centres médicaux $(\mathrm{n}=2 ; 0,95 \%)$ et les cabinets d'accouchement $(n=1 ; 0,47 \%)$. Enfin, les analyses spatiales faites ont permis de montrer que la prévalence palustre clinique est plus élevée (hot spots) dans les quartiers de la commune de Libreville que dans les quartiers des deux autres communes voisines que sont Owendo et Akanda. Les résultats obtenus concourent à faire connaitre la distribution spatiale des formations sanitaires et des hot spots du paludisme dans la région de Libreville-Owendo-Akanda.

Mots-clés: Spatialisation, Morbidité palustre, Structures sanitaires, LibrevilleOwendo-Akanda, Gabon 


\title{
Geography of Malaria in the Region of Libreville-Owendo-Akanda, Gabon
}

\author{
Alexis Mbouloungou, \\ Laboratoire de Géomatique, de Recherche Appliquée et de Conseil \\ (LAGRAC), Université Omar Bongo (UOB), Faculté des Lettres et Sciences \\ Humaines (FLSH), Libreville, Gabon \\ Aubin Armel Koumba, \\ Laboratoire d'Ecologie Vectorielle (LEV), Institut de Recherche en Ecologie \\ Tropicale (IRET), Centre National de la Recherche Scientifique et \\ Technologique, Libreville, Gabon \\ Faculté des Sciences et Techniques (FAST), \\ Université d'Abomey-Calavi, Cotonou, Bénin

\section{Jean Bernard Mombo,} \\ Laboratoire d'Analyse Spatiale et des Environnements Tropicaux \\ (LANASPET), Université Omar Bongo (UOB), Faculté des Lettres et \\ Sciences Humaines (FLSH), Libreville, Gabon

\section{Nadine Ndonghan Iyangui,} \\ Laboratoire de Géomatique, de Recherche Appliquée et de Conseil \\ (LAGRAC), Université Omar Bongo (UOB), Faculté des Lettres et Sciences \\ Humaines (FLSH), Libreville, Gabon \\ Jacques François Mavoungou,
}

Laboratoire d'Ecologie Vectorielle (LEV), Institut de Recherche en Ecologie

Tropicale (IRET), Centre National de la Recherche Scientifique et

Technologique, Libreville, Gabon

Jules Djeki,

Laboratoire de Géomatique, de Recherche Appliquée et de Conseil

(LAGRAC), Université Omar Bongo (UOB), Faculté des Lettres et Sciences

Humaines (FLSH), Libreville, Gabon

\begin{abstract}
In order to determine the geography of malaria, to know the spatial distribution of health facilities and to identify malaria risk areas in LibrevilleOwendo-Akanda area, a study was conducted from july 2016 to june 2017. This study consisted of collecting hospital morbidity data and characterizing public and private health structures in the study area. The malaria data were collected in the clinics using the pre-designed and tested collection forms.
\end{abstract}


Moreover, the cartography of the health structures and chiefdoms of the different neighborhoods from which each patient came was made using a GPSMAP 62s (Garmin). The spatial analyzes were done in ArcGIS 10.3 software according to the Hot Spot model, to identify areas at risk of malaria. Thus, 211 private and public health structures divided into 12 types were identified. These structures are unequally distributed in the LibrevilleOwendo-Akanda region. The best represented are the private medical surgeries $(n=62 ; 29.38 \%)$, the drugstores $(n=39 ; 18.48 \%)$, the structure for nursing treatment $(\mathrm{n}=36 ; 17.06 \%)$. On the other hand, the least represented are the medical centers $(n=2 ; 0.95 \%)$ and childbirth clinics $(n=1 ; 0.47 \%)$. Finally, the spatial analyzes made it possible to show that the clinical malaria prevalence is higher (hot spots) in the districts of the commune of Libreville than in the neighborhoods of the two other neighboring communes that are Owendo and Akanda. The results obtained contribute to make known the spatial distribution of health facilities and hot spots of malaria in the area of Libreville-Owendo-Akanda.

Keywords : Spatialization, Malaria morbidity, Health structures, LibrevilleOwendo-Akanda, Gabon

\section{Introduction}

Le paludisme est la maladie parasitaire la plus répandue dans le monde (Bkhache, 2016 ; Labbé et al., 2017). Cette maladie endémique potentiellement mortelle, due à des parasites Hemococcidae du genre Plasmodium $(P$.$) , est transmise à l'homme par des piqûres des femelles$ d'Anopheles (An.) infectés (Carnevale et al., 2010 ; Pierrat, 2010). Environ 300 à 500 millions de personnes sont touchés chaque année à travers le monde, avec 3 millions de décès par an (Obame-Engonga et al., 2017).

L'Afrique est le continent le plus touché avec environ $92 \%$ des cas de paludisme dont 93\% des décès en 2017 (WHO, 2018). Cette parasitose constitue encore un problème de santé publique majeur en Afrique subsaharienne, où 1 enfant meurt toutes les 30 secondes du paludisme (Djogbenou, 2009 ; Tia et al., 2016).

Cinq espèces plasmodiales sont responsables du paludisme humain dans ce continent : Plasmodium falciparum, $P$. vivax, $P$. malariae, $P$. ovale et P. knowlesi (Maghendji-Nzondo et al., 2016a ; WHO, 2018). De toutes ces espèces de Plasmodium, $P$. falciparum est la plus répandue et la plus virulente avec les formes les plus graves d'accès palustres (Maghendji-Nzondo et al., 2016b ; WHO, 2018). La transmission de ces protozoaires intracellulaires y est assurée principalement par Anopheles gambiae s.l. et An. funestus s.l. (Baska Toussia, 2013 ; Kone et al., 2013). 
Au Gabon, pays d'Afrique centrale, le paludisme est hyper endémique (Maghendji-Nzondo et al., 2016a) et dû principalement à Plasmodium falciparum (Bouyou-Akotet et al., 2012).

Les vecteurs majeurs du paludisme identifiés dans les quartiers sont An. gambiae s.l. et An. funestus s.l. (Mourou et al., 2010 ; Koumba et al., 2019). D'après le Programme National de Lutte contre le Paludisme (PNLP), le paludisme est responsable de la consultation médicale de $45 \%$ des enfants et $71 \%$ des femmes enceintes au Gabon (PNLP, 2015 ; Koumba et al., 2019). De plus, le paludisme demeure la première cause de fréquentation des formations sanitaires, de décès et d'absentéisme aux niveaux scolaire et professionnel. Il est aussi responsable des complications prénatales et infantojuvéniles (DGS-EDS, 2013 ; Ministère de la Santé-SNIS, 2017).

Pour mieux connaitre l'épidémiologie de cette parasitose, diverses études ont été menées dans le pays, tant sur la faune anophélienne que sur le parasite et l'hôte (Mourou et al., 2012 ; Bouyou-Akotet et al., 2014 ; Koumba et al., 2018a, b). Mais, peu de données existe sur la géographie de cette maladie meurtrière. Pourtant, la connaissance et le suivi de la distribution spatiale des données de santé publique sont devenus une nécessité pour la planification (Ndonghan Iyangui, 2014). La présente étude vise à déterminer la distribution spatiale des structures de santé et celle de la prévalence palustre dans la région sanitaire de Libreville-Owendo-Akanda au Gabon.

\section{Materiel et Methodes}

\subsection{Présentation de la région de Libreville-Owendo-Akanda}

La région d'étude regroupe les communes de Libreville, d'Owendo et d'Akanda (Figure 1, ci-après). Elle est limitée au nord et nord-est par la commune d'Akanda, à l'est par l'estuaire de la Mondah, et la commune de Ntoum, et au sud par l'estuaire du Komo et à l'ouest par l'Océan Atlantique.

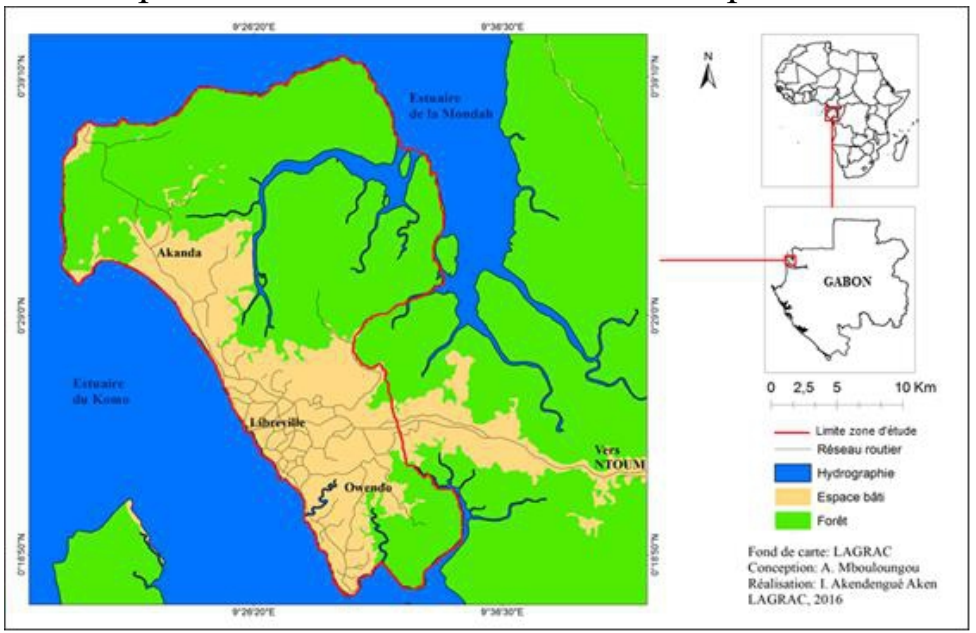

Figure 1: Localisation géographique de la région de Libreville-Owendo-Akanda 
Estimée à 807095 habitants (Pottier et al., 2017), sa population en fait la région la plus peuplée du Gabon (DGS-RGPL, 2015), avec plus de 40\% de la population totale du pays (Bouyou-Akotet et al., 2012).

La région de Libreville-Owendo-Akanda s'insère dans un contexte environnemental de forêts classées contiguës à un espace habité, dont les points culminants sont d'une centaine de mètres d'altitude (Figure 2, ci-après). Ainsi, les communes de Libreville, d'Owendo et d'Akanda sont aménagées sur un ensemble oro-hydrographique accidenté, parsemé de multiples collines et de vallons traversés par des cours d'eau qui se jettent dans l'estuaire du fleuve Komo ou estuaire du Gabon (Ndong Mba, 2004; Mombo \& Itongo, 2011 ; Ndonghan Iyangui, 2016).

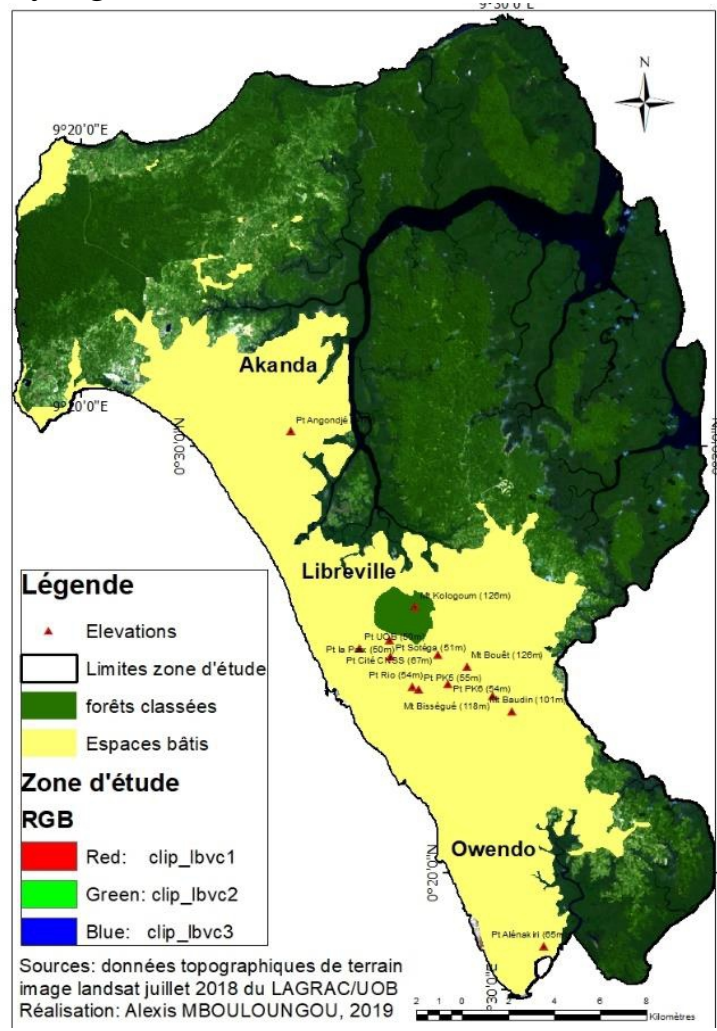

Figure 2: Contexte environnemental de la région de Libreville-Owendo-Akanda

Les principales rivières urbaines sont Gué-Gué, Awondo, Arambo, Batavéa et Lowé (Mombo \& Itongo, 2011). La biodiversité (faune et flore) est riche et diversifiée à cause de la proximité avec le réseau hydrographique dense, les forêts et les mangroves de l'estuaire du Komo et le Parc National d'Akanda (Vande Weghe, 2011 ; ANPN, 2016).

Une étendue de forêt jouxte la région de Libreville-Owendo-Akanda. Ses prolongements sont visibles à Ondogo ou Mindoubé dans la commune de Libreville. Des ilots forestiers intra-urbains, notamment l'Arboretum de 
Sibang dans Libreville et l'Arboretum Raponda Walker dans Akanda, ou la forêt classée de l'Ikoy-Komo, y sont également présents (ANPN, 2016).

La région est soumise à un climat de type équatorial de transition, avec une saison sèche de trois mois (juillet-août-septembre) et une longue saison pluvieuse de neuf mois, marquée par des orages fréquents et des pluies abondantes d'octobre à juin (Maloba Makanga, 2010). D'après la classification de Köppen, la région de Libreville-Owendo-Akanda a un climat tropical de savane. Sa température moyenne annuelle est de $26,3^{\circ} \mathrm{C}$ et ses précipitations sont en moyenne de 1970,6 mm par an (DGMN, 2018). Le mois de juillet est le plus sec, avec $14 \mathrm{~mm}$ de précipitations, tandis qu'octobre est le mois le plus pluvieux avec $307 \mathrm{~mm}$ de pluies.

Globalement, la région d'étude totalise 162 quartiers, soit: 130 quartiers à Libreville, 17 à Owendo et 15 à Akanda. Mais, la difficulté est que tous ces quartiers n'ont pas de limites formelles et matérialisées sur le terrain. Toutefois, leurs chefferies sont clairement identifiées sur le terrain et ont servi de représentation des quartiers et de points de repères, pour la géolocalisation des grappes de cas du paludisme.

\subsection{Collecte des données}

Les données collectées concernent la typologie et les coordonnées géographiques de toutes les structures sanitaires publiques et privées des prestations de soins de santé primaire, de la région d'étude, et les cas de paludisme par unité de santé.

Les points GPS concernant la localisation des structures de santé ont été pris sur le terrain au moyen de l'appareil GPSMAP 64s de marque Garmin. Par contre, les données sur la prévalence palustre, de juillet 2016 à juin 2017, et les caractéristiques de ces structures de santé, ont été récoltées en utilisant des fiches de collecte pré-élaborées et préalablement testées, ainsi que les registres de consultations médicales de chaque unité de santé visitée. La localisation des cas de paludisme s'est faite sur la base des chefferies car, elles représentent l'ensemble des quartiers identifiés dans la région de LibrevilleOwendo-Akanda. Ce choix se justifie par l'absence des limites de chaque unité spatiale urbaine.

Cependant, au cours de cette étude, certaines structures de santé n'avaient pas de registres de consultation bien renseignés, et d'autres avaient des registres dégradés et inexploitables. Aussi, pour l'exploitation des données collectées, seules les formations sanitaires présentant des registres en bon état et complets ont été retenues. Certains établissements sanitaires spécialisés (cabinets dentaires, de rééducation fonctionnelle, de radiologie, d'ophtalmologie, etc.) n'ont pas été pris en compte, car ils ne prennent pas en charge les cas de paludisme. 


\subsection{Analyse spatiale des données}

L'analyse spatiale de la prévalence palustre s'appuie sur le modèle des «points chauds» (Hot spots), qui d'observer les zones les plus impaludées dans la région de Libreville-Owendo-Akanda. Ce modèle permet aussi de déterminer les quartiers à risques palustres. Cet outil devrait aider les services de lutte antivectorielle à mieux cibler leurs actions, dans le sens de prévenir et de réduire la prévalence due à cette parasitose (Chouto \& Wakponou, 2016).

\subsection{Analyse statistique des données}

Les données collectées ont été enregistrées dans une base de données Access 2013 et saisies dans le logiciel Epi Info 7, au regard du volume important d'informations obtenues. Ces données ont été ensuite analysées avec le logiciel $R$ 3.4.4. L'élaboration des cartes a été faite avec celui de cartographie ArcGIS 10.3. Les effectifs, les pourcentages des structures de santé et ceux liés aux cas de paludisme ont été calculés dans Excel 2013. De plus, les comparaisons des pourcentages des cas de paludisme dans les différentes chefferies ont été effectuées à partir du test du Khi 2. Ce test a été considéré comme statistiquement significatif, si la $p$-value est inférieure à 0,05. Les deux fichiers Excel contenant les données de prévalence palustre et les données géographiques ont été fusionnés et importés dans ArcGIS 10.3 (ArcCatalog et ArcMap), pour constituer le Système d'Information Géographique (SIG). Le logiciel ArcGIS 10.3 a été utilisé pour faire des analyses spatiales, à l'aide du modèle des Hots Spots, et élaborer des cartes thématiques.

\section{Resultats}

\subsection{Typologie des structures de santé traitant les cas palustres}

Au cours de cette étude, ont été enregistrées 211 structures médicales et paramédicales qui prennent en charge les cas de paludisme dans la région de Libreville-Owendo-Akanda. Les structures de santé du secteur privé sont les plus nombreuses $(80,57 \% ; \mathrm{n}=170)$, par rapport à celles du secteur public $(19,43 \% ; n=41)$ (Figure 3, ci-après). 


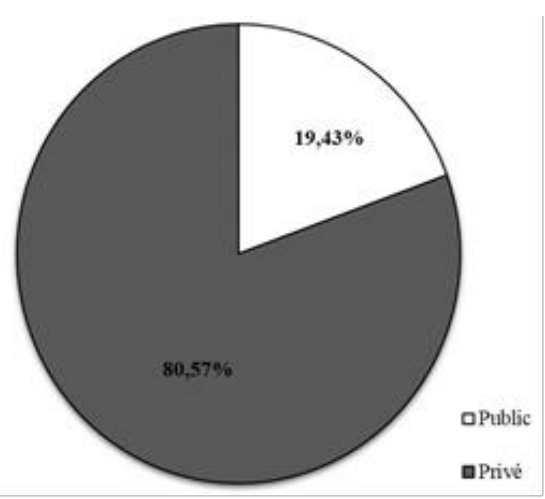

Figure 3: Répartition des structures sanitaires publiques et privées dans la région de Libreville-Owendo-Akanda (Mbouloungou \& Koumba, enquête de terrain 2017)

Par ailleurs, ces 211 structures de santé sont réparties en 12 types. Les types les mieux représentés sont les cabinets médicaux privés $(\mathrm{n}=62$; $29,38 \%)$, les infirmeries $(\mathrm{n}=39 ; 18,48 \%)$, les pharmacies annexées aux cabinets de soins de santé $(\mathrm{n}=36 ; 17,06 \%)$ et les cliniques médicales $(\mathrm{n}=25$; $11,85 \%$ ) (Figure 4, ci-après).

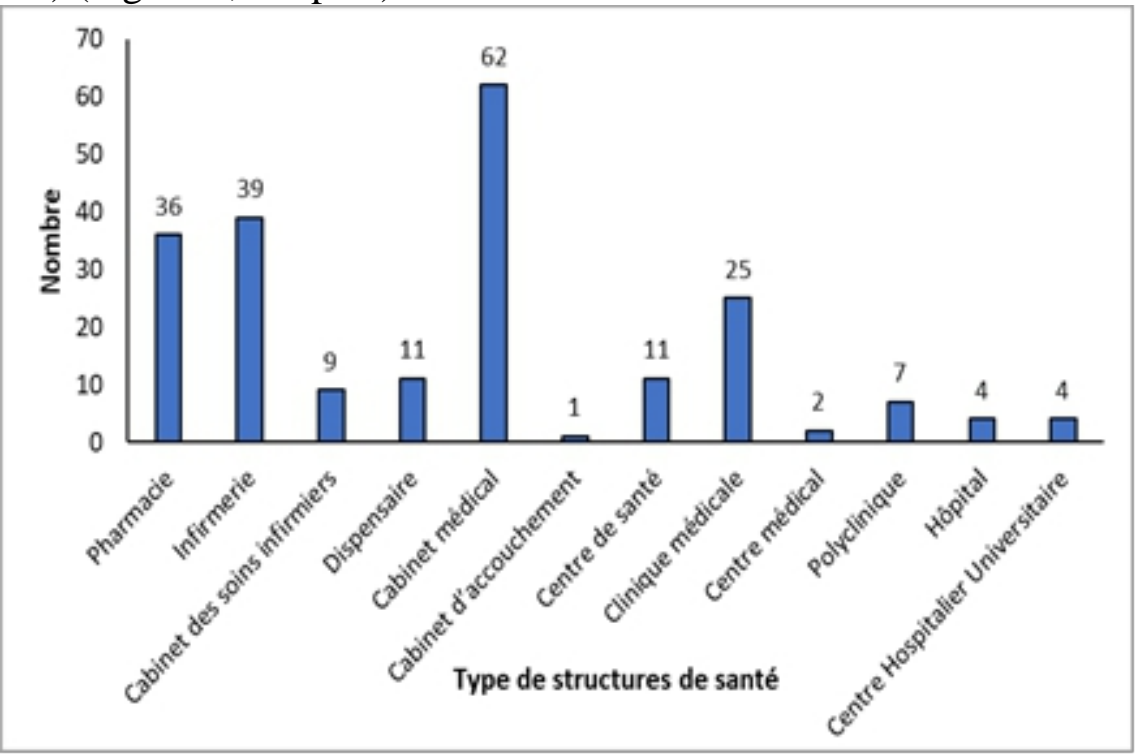

Figure 4: Différents types de structures de santé publiques et privées dans la région de Libreville-Owendo-Akanda (Mbouloungou \& Koumba, enquête de terrain 2017)

Par contre, les types de structures les moins représentés ont été les dispensaires $(\mathrm{n}=11 ; 5,21 \%)$, les centres de santé $(\mathrm{n}=11 ; 5,21 \%)$, les cabinets des soins infirmiers $(\mathrm{n}=9$ : 4,27\%), les polycliniques $(\mathrm{n}=7 ; 3,32 \%)$, les hôpitaux publics ( $\mathrm{n}=4 ; 1,9 \%)$, les centres hospitaliers universitaires ou $\mathrm{CHU}$ $(\mathrm{n}=4 ; 1,9 \%)$, les centres médicaux $(\mathrm{n}=2 ; 0,95 \%)$ et les cabinets d'accouchement ( $\mathrm{n}=1 ; 0,47 \%)$ (Figure 4, ci-avant). 


\subsection{Répartition des structures de santé dans la région de Libreville- Owendo-Akanda}

Le tableau 1, ci-après, montre la repartition spatiale des différentes structures médicales et paramédicales dans les arrondissements des communes de Libreville, Owendo et Akanda. L'analyse des résultats du Tableau 1 et de la Figure 5, ci-après, révèle que les structures de santé sont inégalement réparties dans la région d'étude.

Tableau 1 : Répartition des structures de santé dans la région de Libreville-Owendo-Akanda.

\begin{tabular}{|c|c|c|c|c|c|c|c|c|c|c|c|c|c|c|c|}
\hline t: & 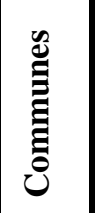 & 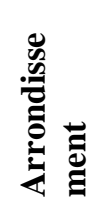 & & 苞 & 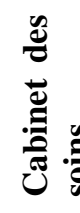 & 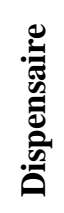 & 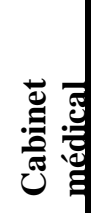 & 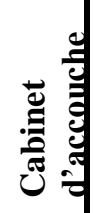 & 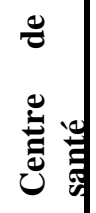 & 莺券 & 章苟 & 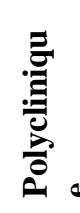 & 产 & 这 & है \\
\hline 1 & \multirow{6}{*}{ 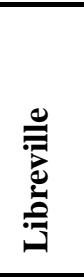 } & $1^{\text {er }}$ & 6 & 7 & 3 & 1 & 5 & 0 & 2 & 5 & 1 & 2 & 0 & 0 & 32 \\
\hline 2 & & $2^{\text {ème }}$ & 5 & 5 & 4 & 0 & 16 & 1 & 1 & 2 & 1 & 1 & 2 & 0 & 38 \\
\hline 3 & & $3^{\text {ème }}$ & 10 & 6 & 0 & 3 & 18 & 0 & 3 & 6 & 0 & 4 & 1 & 1 & 52 \\
\hline 4 & & $4^{\text {ème }}$ & 6 & 3 & 1 & 0 & 15 & 0 & 2 & 8 & 0 & 0 & 0 & 0 & 35 \\
\hline 5 & & $5^{\text {ème }}$ & 2 & 3 & 0 & 0 & 2 & 0 & 1 & 1 & 0 & 0 & 1 & 0 & 10 \\
\hline 6 & & $\boldsymbol{6}^{\text {ème }}$ & 5 & 3 & 1 & 0 & 6 & 0 & 1 & 2 & 0 & 0 & 0 & 1 & 19 \\
\hline 7 & \multirow{2}{*}{ 竞 } & $\mathbf{1}^{\text {er }}$ & 2 & 4 & 0 & 5 & 0 & 0 & 1 & 0 & 0 & 0 & 0 & 1 & 13 \\
\hline 8 & & $\mathbf{2}^{\text {ème }}$ & 0 & 1 & 0 & 1 & 0 & 0 & 0 & 0 & 0 & 0 & 0 & 0 & 2 \\
\hline 9 & \multirow{2}{*}{ 鹃 } & $1^{\text {er }}$ & 0 & 2 & 0 & 1 & 0 & 0 & 0 & 0 & 0 & 0 & 0 & 0 & 3 \\
\hline 10 & & $2^{\text {èe }}$ & 0 & 5 & 0 & 0 & 0 & 0 & 0 & 1 & 0 & 0 & 0 & 1 & 7 \\
\hline Tot & & & 36 & 39 & 9 & 11 & 62 & 1 & 11 & 25 & 2 & 7 & 4 & 4 & 211 \\
\hline
\end{tabular}

Source: Mbouloungou \& Koumba, enquête de terrain 201

Par ailleurs, les arrondissements de la commune de Libreville sont majoritairement les mieux nantis en matière de structures sanitaires (publiques et privés) avec au total 186 structures $(88,15 \%)$, contre $15(7,11 \%)$ et 10 $(4,74 \%)$ formations de santé, respectivement pour les arrondissements d'Owendo et d'Akanda. Le maximum de structures de santé a été enregistré dans le 3ème arrondissement de Libreville ( $\mathrm{n}=52 ; 24,6 \%)$, le 2ème arrondissement $(n=38 ; 18 \%)$, le 4ème arrondissement $(n=35 ; 17 \%)$ et le 1er arrondissement $(n=32 ; 15 \%)$. Par contre, ces structures de soins de santé sont faiblement représentées dans le 6ème arrondissement $(\mathrm{n}=19 ; 10 \%)$ et le 5ème arrondissement ( $\mathrm{n}=10 ; 4,7 \%)$ de Libreville 


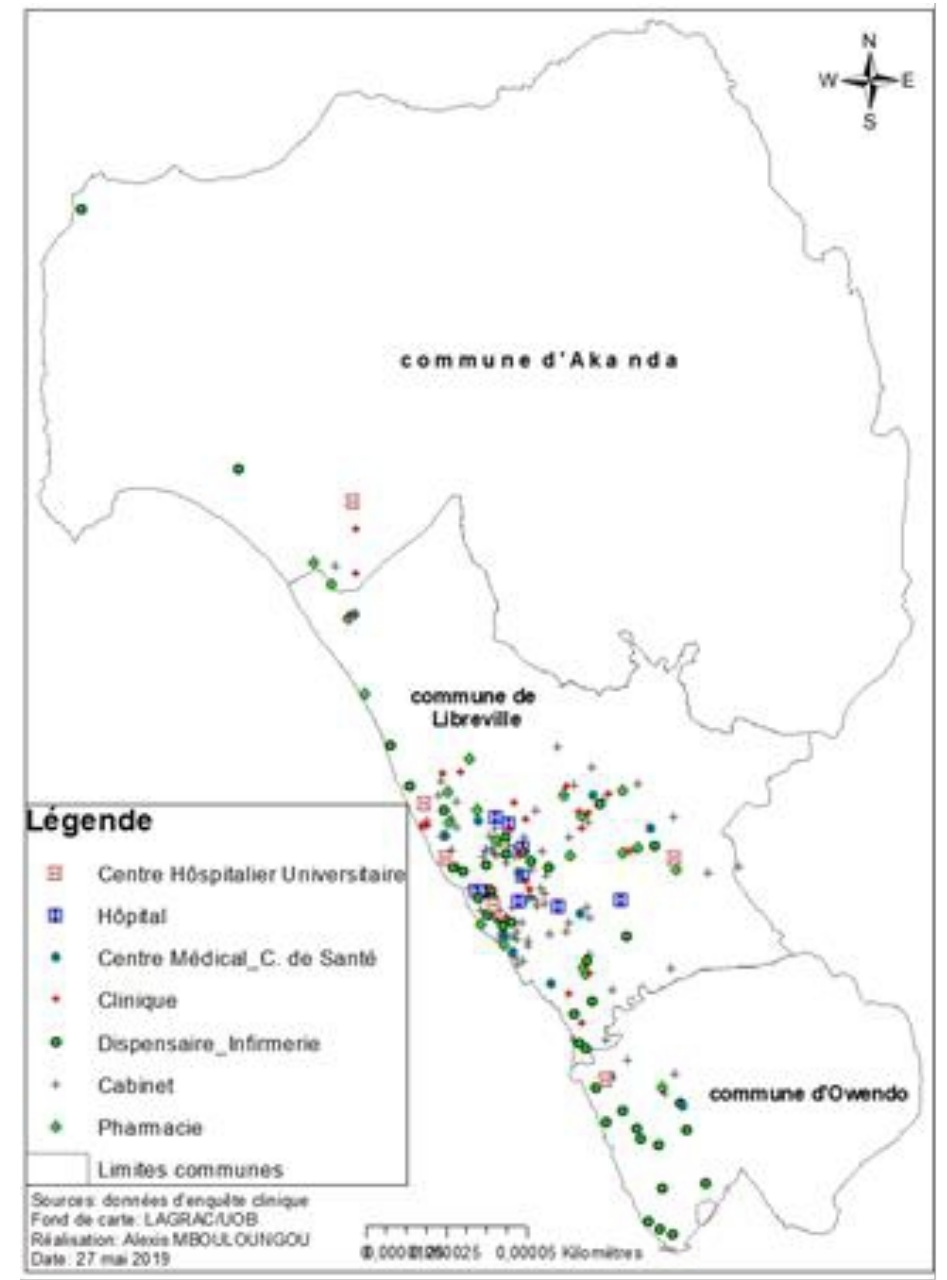

Figure 5: Distribution spatiale des établissements sanitaires de prise en charge du paludisme dans la région d'étude (Mbouloungou \& Koumba, enquête de terrain 2017)

$\mathrm{Au}$ niveau des communes d'Owendo et d'Akanda, le $1^{\text {er }}$ arrondissement d'Owendo $(\mathrm{n}=13 ; 6,16 \%)$ et le $2^{\text {ème }}$ arrondissement d'Akanda $(\mathrm{n}=7 ; 3,32 \%)$ ont quelques structures de santé. Par contre, le $1^{\text {er }}$ arrondissement d'Akanda $(\mathrm{n}=3 ; 1,42 \%)$ et le $2^{\text {ème }}$ arrondissement d'Owendo $(n=2 ; 0,95 \%)$ sont sous-équipés en structures sanitaires.

\subsection{Détermination de la prévalence palustre totale dans la région de Libreville-Owendo-Akanda}

Parmi les 211 structures sanitaires inventoriées, seules 20 établissements de santé ont présenté des registres de consultations en bon état et exploitables. La synthèse des données collectées a permis de recenser au total 17585 malades de paludisme entre le $1^{\text {er }}$ juillet 2016 et le 30 juin 2017, dont 9990 femmes $(56,81 \%)$ et 7595 hommes $(43,19 \%)$ (Figure 6, ci-après). 


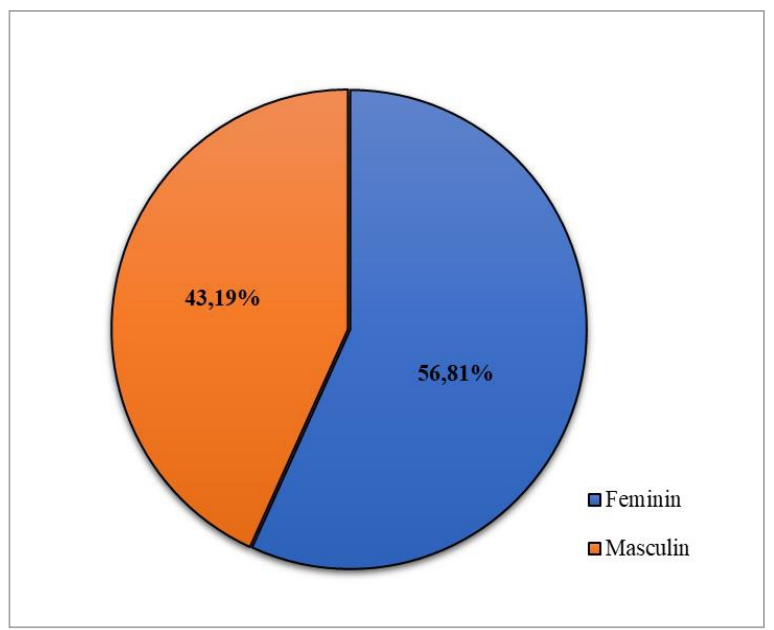

Figure 6: La prévalence palustre selon le genre dans les structures de santé de la région de Libreville-Owendo-Akanda

(Source: Mbouloungou \& Koumba, enquête de terrain 2017)

Tous les patients consultés pour cause de paludisme étaient en moyenne âgés de 16 ans (Tableau 2, ci-après). Cependant, les patients impaludés de sexe féminin étaient plus âgés (19 ans) que ceux de sexe masculin (12 ans). Cette différence d'âge entre les personnes impaludées des deux sexes était statistiquement significative $(\mathrm{p}=0,00)$.

Tableau 2 : Répartition des malades du paludisme par âge moyen et par sexe

\begin{tabular}{|l|l|l|l|}
\hline Sexe & Age moyen $(\mathbf{E T})$ & Intervalle & $\boldsymbol{P}$ \\
\hline Féminin & 19 ans $( \pm 17$ ans $)$ & 1 an -91 ans & \multirow{2}{*}{$\mathrm{P}=0,000$} \\
\hline Masculin & 12 ans $( \pm 14$ ans $)$ & 1 an -76 ans & \\
\hline Les deux sexes & 16 ans $( \pm 16$ ans $)$ & 1 an -91 ans & \\
\hline
\end{tabular}

Source: Mbouloungou \& Koumba, enquête de terrain 2017

\subsection{Distribution spatiale de la prévalence palustre dans la région de Libreville-Owendo-Akanda}

Comme les structures sanitaires, la répartition des cas de paludisme (prévalence palustre) est également hétérogène et inégale au niveau des formations sanitaires. Le choix d'une structure de santé par les malades impaludés est souvent guidé par plusieurs critères, notamment la proximité, la préférence et bien d'autres aspects. La Figure 7, ci-après, présente la répartition des cas de paludisme par structure de santé. 


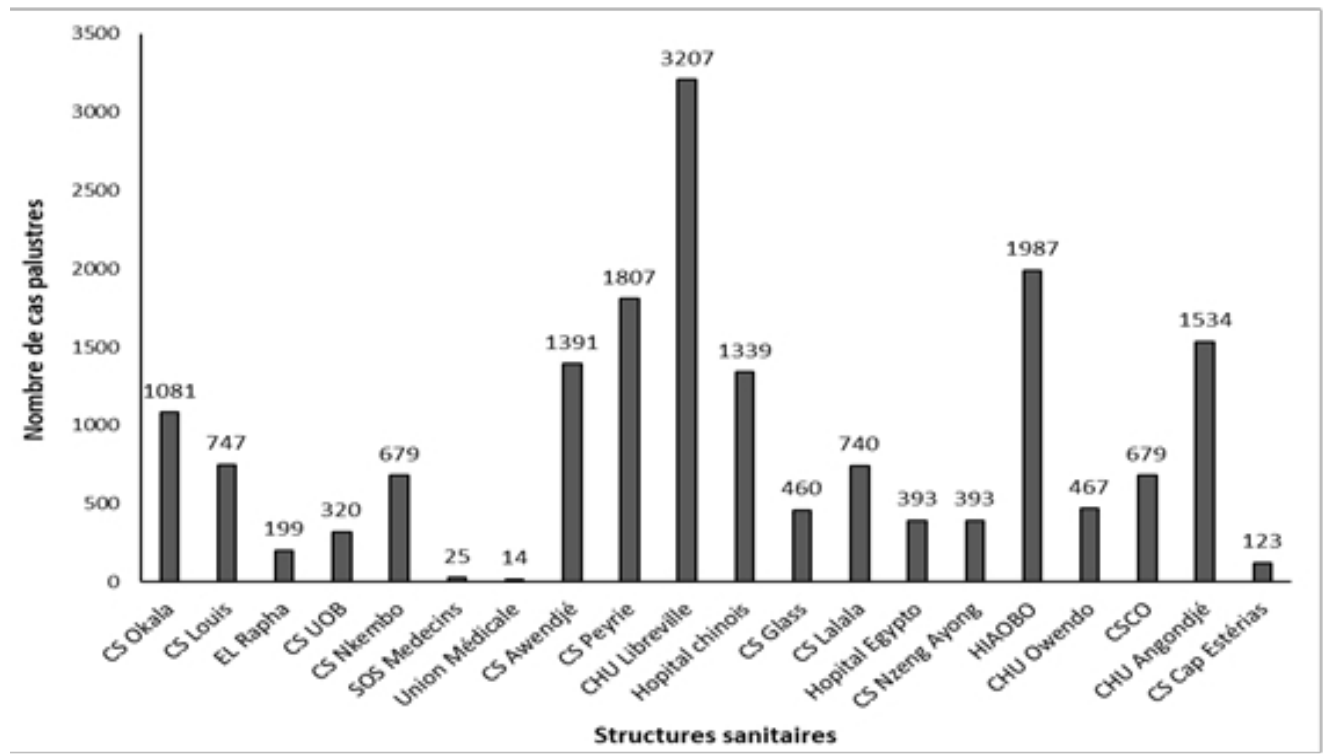

Figure 7: Distribution des cas de paludisme au niveau des structures sanitaires de la région de Libreville-Owendo-Akanda entre juillet 2016 et juin 2017

(Source : Mbouloungou \& Koumba, enquête de terrain 2017)

$\boldsymbol{C H U}$ : Centre Hospitalier Universitaire; CS : Centre de Santé ; CSCO : Centre de Santé Communautaire d'Owendo; HIAOBO : Hôpital des Instructions des Armées Omar Bongo Ondimba ; UOB : Université Omar Bongo.

Le CHU de Libreville (ex-Hôpital Général) est la structure de santé qui a présenté le plus grand nombre de cas de paludisme $(\mathrm{n}=3207 ; 18,24 \%)$. Il est suivi de l'Hôpital des Instructions des Armées Omar Bongo Ondimba (HIAOBO) avec 1987 cas palustres (11,30\%), du Centre de santé de la Peyrie avec 1807 cas de paludisme (10,28\%), du CHU d'Angondjé ( $\mathrm{n}=1534$; 8,72\%), du Centre de santé d'Awendjé ( $\mathrm{n}=1391 ; 7,91 \%$ ), de l'Hôpital de la coopération sino-gabonaise communément appelé Hôpital chinois $(\mathrm{n}=1339$; 7,61\%) et du Centre de santé d'Okala ( $\mathrm{n}=1081 ; 6,15 \%)$.

Les autres formations sanitaires ont enregistré moins de 800 cas de paludisme (5\%), durant la période de réalisation de la présente étude.

\subsection{Détermination des zones à risque palustre dans la région de Libreville-Owendo-Akanda}

Les résultats de l'analyse spatiale font ressortir que les quartiers centraux de la commune de Libreville correspondent aux Hot spots et, donc, les populations de ces quartiers sont plus exposées au paludisme que celles des quartiers des communes d'Akanda et d'Owendo (Figure 8, ci-après). 


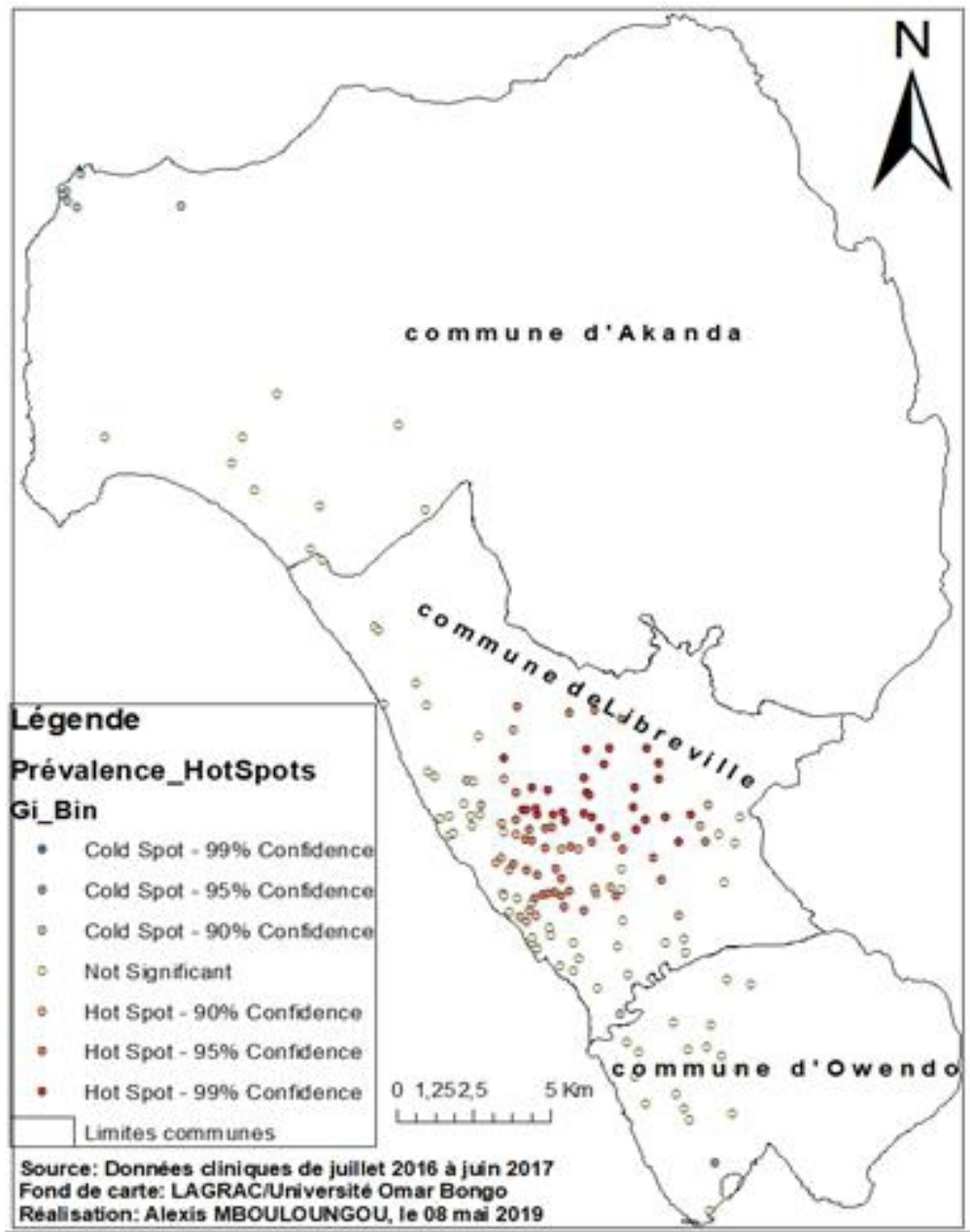

Figure 8: Dispersion spatiale des zones à risque palustre dans la région de LibrevilleOwendo-Akanda (Source: Mbouloungou \& Koumba, enquête de terrain 2017)

Ces quartiers à haut risque palustre sont localisés dans le $2^{\text {ème }}$, le $3^{\text {ème }}$, le $4^{\text {ème }}$ arrondissement de Libreville, une partie du $5^{\text {ème }}$ et $\mathrm{du} 6^{\text {ème }}$ arrondissement de Libreville. En effet, les points les plus chauds (hots spot) concernant le paludisme clinique ont été observés dans ces espaces qui se trouvent au centre de la zone d'étude.

Exceptés ces quartiers hots spots, les autres quartiers sont dits « Cold spot (points froids) » pour le paludisme; ils correspondent aux quartiers faiblement impaludés. Les points froids pour le paludisme sont les quartiers des arrondissements d'Akanda et d'Owendo qui, sont majoritairement intégrés au tissu urbain. 


\section{Discussion}

L'analyse spatiale des structures de santé et des cas de paludisme, qui vient d'être faite dans la région de Libreville-Owendo-Akanda, n'est pas la première du genre. Elle vient compléter les travaux de Ndonghan Iyangui (2014) sur la cartographie de l'offre de soins aux $2^{\text {ème }}$ et $3^{\text {ème }}$ arrondissement de la commune de Libreville. Ce travail a permis de répertorier globalement 211 structures de santé dans la région d'étude. Mais, ces établissements sanitaires sont essentiellement dominés par les structures du secteur privé lucratif. Ce résultat est en accord avec celui obtenu par Ndonghan Iyangui (2014) au niveau des $2^{\text {ème }}$ et $3^{\text {ème }}$ arrondissement de Libreville.

Les structures de santé inventoriées sont distribuées de manière inégale dans la région de Libreville-Owendo-Akanda. En effet, le maximum de structures de santé a été enregistré dans le $3^{\text {ème }}$ arrondissement $(24,6 \%)$, le $2^{\text {ème }}$ arrondissement $(18 \%)$, le $4^{\text {ème }}$ arrondissement $(17 \%)$ et le $1^{\text {er }}$ arrondissement (15\%) de la commune de Libreville. En revanche, ces établissements de santé sont faiblement représentés dans le $6^{\text {ème }}$ arrondissement $(10 \%)$ et le $5^{\text {ème }}$ arrondissement $(4,7 \%)$ de Libreville.

$\mathrm{Au}$ niveau d'Owendo et d'Akanda, le $1^{\text {er }}$ arrondissement d'Owendo $(6,16 \%)$ et le $2^{\text {ème }}$ arrondissement d'Akanda (3,32\%) ont quelques structures de santé tandis que, le $1^{\text {er }}$ arrondissement d'Akanda $(1,42 \%)$ et le $2^{\text {ème }}$ arrondissement d'Owendo (0,95\%) sont sous-équipés en structures sanitaires. Cette inégale répartition des établissements de soins médicaux pourrait s'expliquer, d'une part, par la différence démographique entre ces espaces géographiques et, d'autre part, par le fait que la commune d'Owendo créée en 1995 (Nguema, 2005) et celle d'Akanda fondée en 2013 (JO-Gabon, 2014) sont encore jeunes; elles sont la conséquence de l'expansion de Libreville (Nguema, 2014).

Sur les 211 structures de santé recensées, seules 20 avaient des registres exploitables et/ou complets. Ce faible nombre de formations sanitaires avec registres de consultations adéquats serait lié au fait qu'elles ne sont pas contraintes de communiquer les statistiques à leur Ministère de tutelle. Or, en matière de santé publique, c'est une obligation pour les prises des décisions.

De plus, selon le PNLP (2012), seuls les hôpitaux publics possèdent des registres de consultations et établissent les statistiques des actes médicaux.

D'ailleurs, le Système National d'Information Sanitaire (Ministère de la Santé du Gabon-SNIS, 2016) révèle que la plupart des cliniques privées se focalisent essentiellement sur l'exécution des actes médicaux, sans consigner les résultats de leurs activités dans des registres. Aussi, cette situation est à l'origine des lacunes observées au niveau des données de santé, comme celles liées au paludisme, présentées dans le Système National d'Information Sanitaire. En conséquence, les autorités nationales en charge de la santé 
publique devraient mettre en place une stratégie efficace en vue de la collecte de ces données nécessaires pour la surveillance épidémiologique et la prise de décision. De même, le Ministère de la Santé devrait exiger, à toutes les formations sanitaires des secteurs public et privé, la tenue régulière et à jour des registres de consultations, et la communication des statistiques relatives aux consultations et hospitalisations de leurs établissements.

S'agissant de la prévalence palustre enregistrée dans les formations sanitaires visitées, les analyses spatiales ont montré que les points chauds du paludisme se rencontrent dans les quartiers centraux de Libreville (le $2^{\text {ème }}$, le $3^{\text {ème }}$, le $4^{\text {ème }}$ arrondissement, une partie du $5^{\text {ème }}$ et une autre du $6^{\text {ème }}$ arrondissement de Libreville). Ces quartiers, de la commune de Libreville, sont sous-intégrés. Aussi, la forte prévalence palustre qui y a été observée, pourrait être liée à la topographie contrastée (collines et éperons à versants convexes, pentes fortes, vallées marécageuses), aux comportements et activités humaines (constructions anarchiques, encombrement des réseaux de drainage ou des voies d'évacuation des eaux pluviales, non utilisation des moustiquaires imprégnées), et aux mauvaises conditions de vie (habitat précaire, insalubrité, inondations, promiscuité, etc.).

Ces quartiers sous-intégrés constituent des bidonvilles, qui sont installés dans des bas-fonds inondables et espaces marécageux dits zones «non-aedificandi ». Ces unités spatiales urbaines sont souvent caractérisées par le manque d'urbanisation et d'assainissement, puis par l'absence de Schéma Directeur d'Aménagement et d'Urbanisme (SDAU) et de Plans d'Occupation des Sols (POS). Le plus souvent, elles sont aussi surpeuplées et propices à la prolifération des moustiques vecteurs de Plasmodium et d'arbovirus (Pierrat, 2011 ; Itongo et al., 2017). Les populations humaines de ces bidonvilles sont plus exposées au paludisme que d'autres, du fait de nombreux facteurs humains, écologiques et environnementaux (Chouto \& Wakponou, 2016).

En revanche, les «Cold spots (points froids)» du paludisme correspondent aux quartiers se trouvant dans les zones mieux urbanisées, telles que la Sablière ( $1^{\mathrm{er}}$ arrondissement de Libreville), les cités d'Angondjé (2 ${ }^{\mathrm{ème}}$ arrondissement d'Akanda), les cités de la Société Nationale Immobilière (SNI) à Libreville et Owendo. Il en est également de même pour certaines zones intégrées de la ville de Libreville, qui constituent généralement les zones dites « résidentielles ». En effet, dans ces quartiers, l'environnement domestique est assaini et l'habitat est moderne. Par conséquent, très peu de gîtes larvaires d'anophèles y subsistent (Itongo et al., 2017). De toute évidence, le cadre de vie moderne réduit considérablement la densité anophélienne intradomiciliaire (Carnevale et al., 2010 ; Chouto \& Wakponou, 2016).

Les résultats des analyses spatiales ont montré aussi une inégale distribution des cas palustres (points chauds/points froids). Cette répartition 
inégale pourrait être liée à la différence de faciès rencontrés dans la région d'étude. D'après Chouto \& Wakponou (2016), le degré de transmission du paludisme n'est pas toujours uniforme dans une même zone endémique ; il peut différer dans une même localité. Aussi, convient-il d'étudier la problématique du paludisme urbain à l'échelle spatiale la plus fine (quartier) de la ville, afin de mieux comprendre la distribution spatiale de cette maladie, de faire ressortir avec plus de précision les prévalences palustres et d'embrayer sur les problématiques du cadre de vie des populations humaines dans la région de Libreville-Owendo-Akanda.

A cet effet, Baska Toussia (2013) et Kone et al. (2013) soulignent qu'aujourd'hui, avec l'urbanisation galopante et incontrôlée des villes africaines, le paludisme urbain devra être considéré comme un faciès épidémiologique à part entière, au lieu de toujours faire des analyses de problématiques du paludisme à l'échelle des villes ou des districts de santé.

\section{Conclusion}

Cette esquisse de la géographie des formations sanitaires et des cas de paludisme a permis de localiser avec précision les structures de santé dans la région de Libreville-Owendo-Akanda, d'inventorier les types de structures, de collecter et d'analyser les informations ainsi que les données statistiques relatives à la prévalence palustre dans ces établissements de santé.

$\mathrm{Au}$ cours de cette étude, 17585 cas de paludisme ont été enregistrés dans 20 structures de santé de la region de Libreville-Owendo-Akanda, avec environ $56,81 \%$ de femmes et $43,19 \%$ d'hommes. Ces cas palustres étaient inégalement répartis dans la région. Les analyses spatiales faites, sur la base du modèle des Hot spots, ont permis de montrer que les zones à risque palustre (hot spots) sont les quartiers sous-intégrés de la commune de Libreville. L'environnement et le cadre de vie des populations y sont insalubres et favorables à la transmission du paludisme.

Cependant, les données collectées ne concernent pas toutes les formations sanitaires inventoriées dans la région d'étude, car la majorité d'entre elles ne notifie pas les résultats de leurs activités. Aussi, une harmonisation des statistiques sanitaires devrait-elle s'étendre à toutes les structures de santé, afin d'avoir des données de prévalence palustre suffisantes et, par conséquent, d'apprécier l'ampleur de la morbidité réelle due à cette endémie dans la region de Libreville-Owendo-Akanda.

In fine, le Ministère de la Santé devrait rendre obligatoire la collecte des données de santé dans des registres de consultations et d'hospitalisations, et la communication systématique de ces données par les structures sanitaires. L'objectif est de disposer à terme, d'un fichier fiable et à jour de toutes les structures de santé du pays, et de tous les cas de maladies enregistrés dans 
lesdites structures, afin de consolider la base de données du Système National d'Information Sanitaire (SNIS).

\section{References :}

1. ANPN-Agence Nationale des Parcs Nationaux du Gabon (2016). Dynamiques Spatiales et Impacts Environnementaux des Extensions Urbaines dans l'Arc d'Émeraude. UICN, CEM, WWF, Gabon ; 124p.

2. Baska Toussia, D. V. (2013). Analyse géographique des infections paludéennes dans les districts de santé de la région de l'Extrême-Nord (Cameroun). Université de N'Gaoundéré, Thèse de Doctorat, option Géographie de la santé, 456p.

3. Bkhache, M., Tmimi, F. Z., Charafeddine, O., Faraj, C., Failloux, A. B., \& Sarih, M. (2016). First report of L1014F-kdr mutation in Culex pipiens complex from Morocco. Parasites \& Vectors, 9: 644.

4. Bouyou-Akotet, M. K., Mawili-Mboumba, D. P., Kendjo, E., Eyang Ekouma, A., Raouf, O. A., Engohang Allogho, E., \& Kombila, M. (2012). Complicated malaria and other severe febrile illness in a pediatric ward in Libreville, Gabon. BMC Infectious Diseases, 12: 216.

5. Bouyou-Akotet, M. K., Offouga, C. L., Mawili-Mboumba, D. P., Essola, L., Madoungou, B., \& Kombila, M. (2014). Falciparum malaria as an emerging cause of fever in adults living in Gabon, Central Africa. BioMed Research International, 2014: 1-7.

6. Carnevale, P., Toto, J. C., Guibert, P., Keita, M., \& Mangui, S. (2010). Entomological survey and report of a knockdown resistance mutation in the malaria vector Anopheles gambiae from the Republic of Guinea”. Transactions of the Royal Society of Tropical Medicine and Hygiene, 104 (7): 484-489.

7. Chouto, S., \& Wakponou, A. (2016). Disparités spatio-temporelles et prévalence du paludisme à partir des données formelles: cas de Kousséri (extrême Nord du Cameroun). in : Risques et catastrophes en zone soudano-sahélienne du Cameroun : Aléas, Vulnérabilités et Résiliences. Editions: Cheikh Anta Diop. Editors: Kossoumna Liba'a N., Djiangoué B., Wanie C. Mvo, CERAD-ACP, 8 : 225-245.

8. DGMN-Direction Générale de la Météorologie Nationale du Gabon. (2018). Données climatiques de la région de Libreville-OwendoAkanda, 10p.

9. DGS-EDS (Direction Générale de la Statistique du Gabon- Enquête Démographique et de Santé). (2013). Enquête Démographique et de Santé au Gabon-Phase II (EDSG II), 501p.

10. DGS-RGPL (Direction Générale de la Statistique du GabonRecensement Général de la Population et des Logements). (2015). 
Résultats globaux du Recensement Général de la Population et des Logements de 2013 (RGPL, 2013) du Gabon, Libreville, 247p.

11. Djogbenou, L. (2009). Lutte antivectorielle contre le paludisme et résistance des vecteurs aux insecticides en Afrique. Medecine Tropicale, 69: 160-164.

12. Itongo, M. T., Edou, M., \& Mbouloungou, A. (2017). Climat et paludisme à Libreville (Gabon), de 2011 A 2016. Revue de Géographie de l'Université de Ouagadougou, 2(6): 185-202.

13. JO-Gabon (Journal Officiel de la République Gabonaise). (2014). Numéro 189 du 7 au 14 janvier 2014, p. 1663- Ordonnance n`8/2013 du 21 février 2013 portant suppression du Département du Cap, de la Commune du Cap Estérias et création de la Commune d'Akanda.

14. Kone, A. B., Konan, Y. L., Coulibaly, Z. I., Fofana, D., GuindoCoulibaly, N., Diallo, M., Doannio, J. M. C., Ekra, K. D., \& OdehouriKoudou, P. (2013). Evaluation entomologique du risque d'épidémie urbaine de fièvre jaune survenue en 2008 dans le district d'Abidjan, Côte d'Ivoire. Médecine et Santé Tropicales, 23(1): 66-71.

15. Koumba, A. A., Zinga-Koumba, C. R., Mintsa Nguema, R., Comlan, P., Nkone-Asseko, G., Safiou, A. R., Gneingui, M. P., Ketoh Koffivi, G., Djogbenou, L. S., \& Mavoungou, J. F. (2019). Current sensitivity status of Anopheles gambiae (s.1.) (Culicidae) to DDT and pyrethroids in two agricultural sites of Mouila, Gabon. International Journal of Mosquito Research, 6(1): 12-18.

16. Koumba, A. A., Zinga-Koumba, C. R., Mintsa-Nguema, R., Sevidzem, S. L., Djogbenou, L. S., Akono, P. N., Ketoh, G. K., Faye, O., M'batchi, B., \& Mavoungou, J. F. (2018a). Identification of the knockdown resistance (Kdr) mutations in Anopheles gambiae s.l. in the Mouila area, Southwest Gabon. Journal of Entomology and Zoology Studies, 6(3): 602-607.

17. Koumba, A. A., Zinga-Koumba, C. R., Mintsa-Nguema, R., Obame Ondo, P., Bibang Bengono, G., Comlan, P., Ketoh, G. K., Djogbenou, L. S., Faye, O., M'batchi, B., \& Mavoungou, J. F. (2018b). Susceptibilité d'Anopheles gambiae s.s. et An. coluzzii aux organophosphorés et aux carbamates en zones d'exploitation industrielle de palmiers à huile à Mouila, Gabon. Bulletin de la Société de Pathologie Exotique, 111: 176 - 182.

18. Labbé, P., David, J. P., Alout, H., Milesi, P., Djogbenou, L., Pasteur, N., \& Weill, M. (2017). Evolution of resistance to insecticide in disease vectors. Genetics and Evolution of Infectious Diseases, 7: 313339.

19. Maghendji-Nzondo, S., Nzoughe, H., Lemamy, G. J., Kouna, L. C., Pegha-Moukandja, I., Lekoulou, F., Mbatchi, B., Toure-Ndouo, F., \& 
Lekana-Douki, J. B. (2016a). Prevalence of malaria, prevention measures, and main clinical features in febrile children admitted to the Franceville Regional Hospital, Gabon. Parasite, 23 (32): 1-9.

20. Maghendji-Nzondo, S., Kouna, L. C., Mourembou, G., Boundenga, L., Imboumy-Limoukou, R. K., Matsiegui, P. B., Manego-Zoleko, R., Mbatchi, B., Raoult, D., Toure-Ndouo, F., \& Lekana-Douki, J. B. (2016b). Malaria in urban, semi-urban and rural areas of southern of Gabon: comparison of the Pfmdr 1 and Pfcrt genotypes from symptomatic children", Malaria Journal, 15: 420.

21. Maloba-Makanga, J. D. (2010). Les précipitations au Gabon: climatologie analytique en Afrique. Editions Harmattan, Paris, 146p.

22. Ministère de la santé-SNIS (2016). Mission de collecte des donnés en vue d'une cartographie pour établir un état des lieux, des équipements, des usages et perception des TIC des établissements concernés par le Système National d'Information Sanitaire (SNIS). Rapport final de mission, Libreville, 139p.

23. Mombo, J. B., \& Itongo, M. T. (2011). Gestion du site et risques naturels à Libreville. Gabonica (revue du CERGEP), n 5 , pp. 1-21.

24. Mourou, J.-R., Coffinet, T., Jarjaval, F., Pradines, B., Amalvict, R., Rogier, C., Kombila, M., Pagès, F. (2010). Malaria transmission and insecticide resistance of Anopheles gambiae in Libreville and PortGentil, Gabon. Malaria Journal, 9: 321: 1-8.

25. Mourou, J. R., Coffinet, T., Jarjaval, F., Pradines, B., Cotteaux, C., Godefroy, L., Kombila, M., \& Pages, F. (2012). Malaria transmission in Libreville: results of one year survey. Malaria Journal, 11(40): 114.

26. Ndong Mba, J. C. (2004). Libreville, Owendo. Atlas de l'Afrique, pp. 60-61.

27. Ndonghan Iyangui, N. N. (2014). Utilité de l'information géographique sanitaire: exploration cartographique de l'offre de soins à Libreville. Annales de 1'UOB, Presses Universitaires du Gabon, 19: 51-66.

28. Ndonghan Iyangui, N. N. (2016). Proximité des stations de base de téléphonie mobile et risques pour la santé à Libreville (Gabon). Revue Canadienne de Géographie Tropicale/Canadian Journal of Tropical Geography, [En ligne], (3) 1. Mis en ligne le 05 mai 2016, pp. 15-28, consulté le 10 août 2019. URL: http://laurentienne.ca/rcgt

29. Nguema, R.-M. (2005). Développement de la ville, découpage et appropriation des territoires urbains au Gabon : le cas de Libreville », Belgeo, [En ligne], 4. Mis en ligne le 29 octobre 2013, consulté le 15 août 2019. URL : http://journals.openedition.org/belgeo/12167 ; DOI : $10.4000 /$ belgeo. 12167 
30. Nguema, R. M. (2014). Croissance et aménagement de l'agglomération de Libreville. Piste pour une gestion de la ville de demain. Les enjeux et défis du Gabon au $\mathrm{XX}^{\text {ème }}$ siècle, Edition Croissance et savoir, Paris, pp. 101-129.

31. Obame-Engonga, L. C., Sima-Obiang, C., Ngoua-Meye-Misso, R. L., Orango Bourdette, J. O., Ondo, J. P., Ndong Atome, G. R., Koudou, J., \& Traoré, S. A. (2017). Larvicidal and ovicidal properties against Anopheles gambiae, antioxidant and antibacterial activities of the combination of essential oils Eucalyptus citriodora, Cymbopogon giganteus and Cymbopogon nardus from Gabon. Journal of Multidisciplinary Engineering Science and Technology, 4: 78867894.

32. Pierrat, C. (2010). Des moustiques et des hommes : Les territoires du paludisme à Tori-Bossito (Sud du Bénin). Thèse de Doctorat Unique, Université de Paris 1 Panthéon Sorbonne, 185p.

33. Pierrat, C. (2011) Risque palustre : appréhender la vulnérabilité des individus à l'échelle locale (Sud du Bénin) ». VertigO, 11 (3) : 12-29.

34. PNLP-Programme National de Lutte contre le Paludisme. (2012). Plan Stratégique National de lutte contre le paludisme du Gabon 2013-2017. Edition OMS, 106p.

35. PNLP-Programme National de Lutte contre le Paludisme (2015). Revue à mi-parcours du Plan Stratégique National 2013-17 du Programme National de Lutte contre le Paludisme, 98p.

36. Pottier, P., Menie Ovono, Z., Faure, F. E., Bignoumba, G. S. (2017). Les régions littorales du Gabon : Éléments de réflexion pour une planification stratégique du territoire. Programme interuniversitaire Nantes (France)-Libreville (Gabon), 440p.

37. Tia, E., Konan, K. G., Boby Ouassa, A. M., Moussa, K., Tea Sea, A., Koffi, B., \& Kadjo K. (2016). Evaluation au laboratoire de l'efficacité de la peinture insecticide acrylique titan ${ }^{\circledR}$ contre Anopheles gambiae, vecteur majeur du paludisme en Côte d'Ivoire. European Scientific Journal, 12(27) : 229-241.

38. Vande Weghe, J. P. (2011). Les parcs nationaux du Gabon : Akanda et

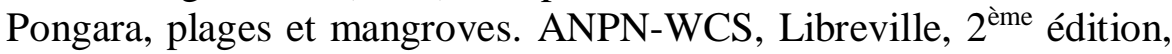
272p.

39. WHO (2018). World Malaria Report 2018. World Health Organization 2018, Geneva, 210p. 